\title{
Author Correction to: In silico studies of novel scaffold of thiazolidin-4-one derivatives as anti-Toxoplasma gondii agents by 2D/3D-QSAR, molecular docking, and molecular dynamics simulations
}

\author{
Rahman Abdizadeh $^{1}$ - Farzin Hadizadeh ${ }^{2} \cdot$ Tooba Abdizadeh $^{3}$ \\ Published online: 14 March 2020 \\ (C) Springer Science+Business Media, LLC, part of Springer Nature 2020
}

Author Correction to: Structural Chemistry

https://doi.org/10.1007/s11224-019-01458-y

The authors regret that the Acknowledgments of the original version of this article was incorrect. The correct Acknowledgments should read:

Acknowledgments We are grateful to Clinical Biochemistry Research Center, Basic Health Sciences Institute, Sharekord University of Medicinal Sciences. The study was supported financially by part of the grant (No. 1394-01-70-2514) provided by Shahrekord University of Medical Sciences and also, was approved by the Ethics Committee of Shahrekord University of Medical Sciences (IR.SKUMS.REC.1394.238). The authors would like to apologize for any inconvenience caused.

The online version of the original article can be found at https://oi.org/ 10.1007/s11224-019-01458-y

Tooba Abdizadeh

t.abdizadeh@gmail.com

1 Department of Medical Parasitology and Mycology, Faculty of Medicine, Shahrekord University of Medical Sciences,

Shahrekord, Iran

2 Biotechnology Research Center, Pharmaceutical Technology Institute, Mashhad University of Medical Sciences, Mashhad, Iran

3 Clinical Biochemistry Research Center, Basic Health Sciences Institute, Shahrekord University of Medical Sciences,

Shahrekord, Iran 